\title{
ASSOCIAÇÃO ENTRE A FORÇA DE PREENSÃO PALMAR E A FORÇA MUSCULAR RESPIRATÓRIA DE JOVENS POR SEXO
}

\author{
ASSOCIATION BETWEEN THE PALMAR PRESSURE FORCE AND \\ THE RESPIRATORY MUSCULAR FORCE OF YOUNG PEOPLE BY \\ SEX
}

\author{
Aurelia Maria Pessoa Silva de Oliveira ${ }^{1}$ \\ Elisangela Vilar de Assis ${ }^{2}$ \\ Pollianna Marys de Souza e Silva ${ }^{3}$ \\ Ubiraídys de Andrade Isidório ${ }^{4}$ \\ Marta Ligia Vieira Melo ${ }^{5}$
}

\begin{abstract}
RESUMO: Objetivo: Avaliar a associação entre a força de preensão palmar e a força muscular respiratória de jovens por sexo. Método: Trata-se de um estudo transversal analítico e quantitativo. A pesquisa foi realizada em uma Clínica Escola Integrada e no laboratório de Fisioterapia durante o mês de abril de 2018. Foram avaliados universitários com idade entre 18 e 35 anos, de ambos os sexos, sendo excluídos, fumantes, os que apresentavam comprometimento da função muscular respiratória e da mão dominante, sobrepeso e/ou obesidade e gestantes. Foram coletados dados pessoais e informações sobre a condição de saúde prévia dos participantes, realizada mensuração da força muscular inspiratória (Pimáx) e expiratória (Pemáx), além da força de preensão palmar. Os participantes foram divididos em dois grupos conforme o sexo. Resultados: Foram avaliados 40 universitários de ambos os sexos, a maioria não apresentou antecedentes pessoais para doenças cardiovasculares $(97,5 \%)$, e $40 \%$ consomem uso de álcool esporadicamente. Observou-se que em relação ao hábito de relaizar atividades

\footnotetext{
${ }^{1}$ Fisioterapeuta graduada pela Faculdade Santa Maria - FSM, Cajazeiras, Paraíba, Brasil.

2 Doutora em Ciências pela Faculdade de Medicina do ABC, Santo André, São Paulo, Brasil. Professora Adjunta da Universidade Federal de Campina Grande - UFCG, Cajazeiras, Paraíba, Brasil.

${ }^{3}$ Doutoranda em Ciência da Informação pela Universidade Federal da Paraíba - UFPB, João Pessoa, Paraíba, Brasil. Professora visitante da Pós - Graduação em Fisioterapia em Neonatal e Pediátrica do Centro Universitário de João Pessoa - UNIPÊ, módulo de prática em Fisioterapia Neonatal, João Pessoa, Paraíba, Brasil.

${ }^{4}$ Doutorando em Ciências da Saúde pela Faculdade de Medicina do ABC, Santo André, São Paulo, Brasil. Professor da Faculdade Santa Maria - FSM, Cajazeiras, Paraíba, Brasil.

${ }^{5}$ Mestre em Saúde Coletiva pela Universidade de Santos - UNISANTOS, Santos, São Paulo. Professora da Faculdade Santa Maria - FSM, Cajazeiras, Paraíba, Brasil.
} 
físicas, uma pequena maioria de mulheres (55\%) não praticavam, enquanto que maioria de homens $(70 \%)$ praticava alguma atividade física. Houve correlação positiva e estaticamente significativa entre Pimáx e Pemáx no sexo feminino e também uma correlação negativa e significativa entre Pimax e FPP no sexo masculino. Conclusão: Observou-se que à medida que a Pimáx aumenta, aumenta também a Pemáx em mulheres. Nos homens foi observado que à medida que a força de preensão palmar aumenta, diminui a Pimáx. Mais estudos são necessários para elucidar essas relações.

Palavras chave: Adulto. Dinamômetro de força muscular. Força muscular respiratória. Preensão palmar.

ABSTRACT: Objective: To evaluate the association between handgrip strength and respiratory muscle strength in young people by sex. Method: This is a crosssectional analytical and quantitative study. The research was carried out in an Integrated School Clinic and in the Physiotherapy laboratory during the month of April 2018. University students aged between 18 and 35 years, of both sexes, were excluded, smokers, those with impaired muscle function respiratory and dominant hand, overweight and / or obesity and pregnant women. Personal data and information on the participants' prior health condition were collected, and inspiratory (Pimax) and expiratory (Pmax) muscle strength measurements were performed, in addition to hand grip strength. Participants were divided into two groups according to sex. Results: 40 university students of both sexes were evaluated, most had no personal history of cardiovascular disease (97.5\%), never smoked (100\%) and 40\% used alcohol sporadically. It was observed that in relation to the practice of physical activities, a small majority of women (55\%) do not practice, while the majority of men (70\%) practice some physical activity. There was a positive and statically significant correlation between Pimax and Pemáx in females and also a negative and significant correlation between Pimax and FPP in males. Conclusion: It was observed that as Pimax increases, so does Pema in women. In men, it was observed that as the handgrip strength increases, the Pimax decreases. Further studies are needed to elucidate these relationships.

Keywords: Adult. Muscle strength dynamometer. Respiratory muscle strength. Hand grip. 


\section{INTRODUÇÃO}

A força muscular é simétrica a quantidade de proteínas contráteis existentes no músculo, onde, são solicitadas durante uma contração. Inúmeros fatores podem intervir na contração muscular, como: sexo, idade e antropometria (MENDES; AZEVEDO; AMARAL, 2013).

A força muscular das mãos é avaliada a partir da aplicação de um esforço isométrico, alcançado durante a execução do movimento de preensão palmar que, juntamente a um músculo ou grupo muscular, determina o estado de força geral do corpo do indivíduo, estabelecendo uma relação prática da integridade funcional dos músculos, sendo esses níveis adequados de força muscular indicativos de saúde global (MOREIRA et al., 2008; DIAS et al., 2010).

Nascimento et al. (2010) descreveram em seus estudos que, entre 25 a 35 anos, a força de preensão palmar (FPP) é mais forte e, após essa idade, há um decréscimo gradual dessa força. Uma diminuição da função muscular poderá comprometer a realização das atividades de vida diárias (AVD's) ocasionando, portanto, um maior impacto na qualidade de vida dos sujeitos (SANTOS et al., 2015).

A diminuição da força e potência muscular pode estar relacionada a um mau funcionamento da força muscular respiratória (FMR) (SIMÕES et al., 2007, VALLE et al., 2012, ALMEIDA; BERTUCCI; LIMA et al., 2008). A redução de FMR é um dos fatores relacionados à intolerância ao exercício que, consequentemente, irá somar com a redução do bem-estar global do indivíduo, diminuindo, assim, a qualidade de vida dos mesmos (BESSA; LOPES; RUFINO, 2015). Segundo Fonseca (2017) uma redução da força dos músculos esqueléticos e dos músculos respiratórios irá ocorrer com o aumento da idade, assim, quanto maior a idade maior será à diminuição da força muscular.

Acredita-se que a FMR e a FPP apresentam uma relação direta ou indireta com a saúde do indivíduo, de modo que, quaisquer alterações em um desses processos contribuirão para o comprometimento no desempenho muscular ou 
respiratório. Partindo desse ponto de vista, se faz necessário o conhecimento prévio dos assuntos, força da musculatura respiratória e a força de preensão palmar, colaborando, dessa forma, para a descoberta de alterações de maneira precoce e, a partir dessas alterações, traçar um plano de tratamento específico, contribuindo para saúde dos indivíduos. Dessa forma, o objetivo desta pesquisa é avaliar a associação entre a FPP e a FMR de jovens por sexo.

\section{MÉTODO}

Trata-se de um estudo transversal analítico e quantitativo.

A coleta ocorreu durante o mês de abril de 2018, de segunda a sexta-feira, das $8 \mathrm{~h}$ às $11 \mathrm{~h}$ e das $13 \mathrm{~h}$ às $17 \mathrm{~h}$, em uma Clínica Escola Integrada e no laboratório de Fisioterapia localizada na cidade de Cajazeiras/PB. A seleção dos participantes ocorreu por conveniência, sendo avaliados 47 universitários com idade entre 18 e 35 anos, de ambos os sexos, devidamente matriculados na instituição, nos turnos matutino e vespertino, sendo excluídos da pesquisa; fumantes, os que apresentaram comprometimento da função muscular respiratória e da mão dominante, que estivessem com sobrepeso e/ou obesidade e gestantes. Participaram da pesquisa 40 alunos de vários cursos da Faculdade.

Para a busca de informações, foi construído pelos pesquisadores um questionário semiestruturado, constando os seguintes ítens: iniciais, cor, idade e sexo, hábitos de vida, tais como: faz uso de tabaco (cigarro) ou consome bebida alcoólica; antecedentes pessoais para doenças cardiovasculares (DCV'S) como: obesidade, hipertensão, diabetes, cardiopatias, e doenças renais; prática de atividade física (auto relatada); avaliação da FMR; avaliação da PFP; avaliação antropométrica (peso e altura para o cálculo do índice de massa corpórea (IMC), circunferência abdominal (CA), circunferência da cintura (CC), circunferência do quadril (CQ), relação cintura quadril) e verificação dos índices pressóricos (pressão arterial sistólica e pressão arterial diastólica). 
Os voluntários foram convidados a participarem da avaliação clínica pelos pesquisadores e orientados a estarem, preferencialmente, de roupas leves e sem calçados. Foi coletada a estatura e a massa corporal dos voluntários, a partir de um estadiômetro e de uma balança analógica com capacidade máxima de $150 \mathrm{~kg}$ e divisão de 100g (SAMPAIO; FIGUEIREDO, 2005). Após a coleta do peso e altura foi calculado o IMC (IMC), este cálculo foi obtido por meio da relação entre a massa corporal da pessoa e a sua altura, que se baseia no seguinte cálculo IMC = peso $(\mathrm{kg}) /$ altura $^{2}(\mathrm{~cm})$. A classificação ou o resultado do IMC foram os preconizados pela Organização Mundial da Saúde (OMS), de modo que os valores são considerados: baixo peso (IMC < 18,5 kg/m²), eutrofia (IMC 18,5-24,99 kg/m²), sobrepeso (IMC 25$29,99 \mathrm{~kg} / \mathrm{m}^{2}$ ), e obesidade (IMC e $\geq 30,00 \mathrm{~kg} / \mathrm{m}^{2}$ ) (REZENDE et al., 2006).

Para a obtenção dos valores da circunferência abdominal (CA) foi utilizada uma fita métrica flexível e que não altera o tamanho, com divisões em centímetros. Esta foi posicionada entre as costelas inferiores e as cristas ilíacas. Os dados coletados foram avaliados, sendo adotados os valores de acordo com o grau de risco para doenças cardiovasculares, para homens a CA > $94 \mathrm{~cm}$ e para mulheres a $\mathrm{CA}>80 \mathrm{~cm}$, são indicativos de riscos para a instalação das doenças cardiovasculares (REZENDE et al., 2006).

A FPP foi coletada por meio do dinamômetro digital da marca CAMRY. Para a avaliação o indivíduo foi posicionado de maneira confortável, sentado com a coluna ereta, o quadril e os joelhos a $90^{\circ}$ de flexão, ombro em adução os cotovelo fletido a $90^{\circ}$, antebraço e punho em posição neutra, juntamente com uma extensão do braço realizando, dessa forma, uma pronação incompleta ou meia pronaçãolsemipronação. A dinamometria de preensão manual foi coletada apenas no membro dominante, efetivando três reproduções de força com um intervalo de repouso de 1 minuto, para que o participante não apresentasse fadiga, sendo assim, adotado o valor maior, enquanto o examinador foi posicionado segurando o dinamômetro (SOARES et al., 2012; EICHINGER et al., 2015 ).

A FMR foi coletada por meio do aparelho manovacuômetro, pelo qual foram mensuradas as pressões respiratórias máximas correspondes a força muscular inspiratória máxima (PImáx) e força muscular expiratória máxima (PEmáx). O 
aparelho é ajustado em cmH2O e com variação de $\pm 300 \mathrm{cmH} 2 \mathrm{O}$ (COSTA et al., 2010).

Para a avaliação o voluntário foi posicionado sentado, coluna ereta, com o tronco a $90^{\circ}$ em relação ao quadril, o nariz dele foi ocluído com clipe nasal durante toda avaliação. Para que não houvesse oscilações dos resultados da PEmáx e PImáx durante o teste, as determinações seriadas foram realizadas na mesma postura, os universitários realizaram três manobras, com sustentações de pelo menos um segundo, e sempre adotando o valor maior. Os procedimentos das manobras adotadas foram os preconizados por Bessa; Lopes; Rufino (2015) e Costa et al. (2010).

Para dar início a coleta de dados o estudo foi submetido ao Comitê de Ética em Pesquisa da Faculdade Santa Maria, após a aprovação nessa instância, foi dado início a a coleta dos dados e os universitários foram convidados a participar da pesquisa por meio da entrevista do questionário semiestruturado e assinatura do Termo de Consentimento Livre e Esclarecido (TCLE). Em todos os passos foram respeitados os protocolos da ética, assegurando os direitos e deveres que dizem respeito à comunidade científica e aos indivíduos envolvidos na pesquisa. Este estudo faz parte da pesquisa intitulada "Associação entre capacidade funcional e física e força muscular esquelética de adultos jovens" que foi aprovado pelo Comitê de Ética em Pesquisa da Faculdade Santa Maria sob o protocolo 2.556.330 (BRASIL, 2012).

Os dados foram analisados no SPSS (versão 25). Além de estatísticas descritivas de frequência absoluta e relativa, medidas de tendência central e de dispersão, utilizou-se correlação de Pearson, com uma significância estatística $p \leq$ 0,05 .

\section{RESULTADOS}

A amostra para este estudo foi composta por 40 estudantes universitários saudáveis de ambos os sexos. 
Na tabela 1 observa-se prevalência de pessoas solteiras, de etnia parda.

Tabela 1 - Descrição sociodemográfica dos dados.

\begin{tabular}{lcccc}
\hline Variáveis & \multicolumn{2}{c}{ Feminino $(\mathbf{n = 2 0})$} & \multicolumn{2}{c}{ Masculino $(\mathbf{n = 2 0})$} \\
\hline & $\begin{array}{c}\text { Frequência } \\
\text { absoluta (F) }\end{array}$ & $\begin{array}{c}\text { Frequência } \\
\text { relativa (\%) }\end{array}$ & $\begin{array}{c}\text { Frequência } \\
\text { absoluta (F) }\end{array}$ & $\begin{array}{c}\text { Frequência } \\
\text { relativa (\%) }\end{array}$ \\
$\begin{array}{l}\text { Estado civil } \\
\text { Solteiro }\end{array}$ & 18 & 90,0 & 19 & \\
Casado & 2 & 10,0 & 1 & 95,0 \\
Etnia & 6 & 30,0 & 5 & 5,0 \\
Branco & 14 & 70,0 & 14 & 25,0 \\
Pardo & 0 & 0,0 & 1 & 70,0 \\
Negro & & &
\end{tabular}

Fonte: Dados da pesquisa 2018.

$\mathrm{Na}$ tabela 2 pode-se observar que estudantes, de ambos os sexos, não tem antecedentes pessoais para doenças cardiovasculares (DCV'S), não fazem uso de tabaco, mas consomem álcool. Em relação à prática de atividades físicas, a maioria de mulheres não pratica (70\%), enquanto a maioria de homens se exercita (45\%).

Tabela 2 - Descrição dos dados relativos à saúde e hábitos de vida.

\begin{tabular}{|c|c|c|c|c|}
\hline \multirow[b]{2}{*}{ Variáveis } & \multicolumn{2}{|c|}{ Feminino $(n=20)$} & \multicolumn{2}{|c|}{ Masculino $(n=20)$} \\
\hline & $\begin{array}{l}\text { Frequência } \\
\text { absoluta (F) }\end{array}$ & $\begin{array}{l}\text { Frequência } \\
\text { relativa (\%) }\end{array}$ & $\begin{array}{l}\text { Frequência } \\
\text { absoluta (F) }\end{array}$ & $\begin{array}{l}\text { Frequência } \\
\text { relativa (\%) }\end{array}$ \\
\hline \multicolumn{5}{|l|}{$\begin{array}{l}\text { Antecedentes } \\
\text { pessoais para } \\
\text { DCV'S }\end{array}$} \\
\hline $\begin{array}{l}\text { Sem } \\
\text { antecedentes }\end{array}$ & 19 & 95,0 & 20 & 100,0 \\
\hline $\begin{array}{l}\text { Doenças } \\
\text { Renais } \\
\text { Álcool }\end{array}$ & 1 & 5,0 & 0 & 0,0 \\
\hline Sim & 7 & 35,0 & 9 & 45,0 \\
\hline Não & 13 & 65,0 & 11 & 55,0 \\
\hline $\begin{array}{l}\text { Frequência } \\
\text { no consumo } \\
\text { de álcool }\end{array}$ & & & & \\
\hline $\begin{array}{l}\text { Esporadicame } \\
\text { nte }\end{array}$ & 4 & 20,0 & 4 & 20,0 \\
\hline Uma vez ao m & 1 & 5,0 & 3 & 15,0 \\
\hline
\end{tabular}




\begin{tabular}{|c|c|c|c|c|}
\hline $\begin{array}{l}\text { Todo fim de } \\
\text { semana } \\
\text { Duas ou mais }\end{array}$ & 2 & 10,0 & 1 & 5,0 \\
\hline $\begin{array}{l}\text { vezes por } \\
\text { semana }\end{array}$ & 0 & 0,0 & 1 & 5,0 \\
\hline $\begin{array}{l}\text { Não consome } \\
\text { Prática de } \\
\text { atividade } \\
\text { física }\end{array}$ & 13 & 65,0 & 11 & 55,0 \\
\hline Sedentário & 11 & 55,0 & 6 & 30,0 \\
\hline $\begin{array}{l}\text { Realiza } \\
\text { atividade } \\
\text { Atividade } \\
\text { física }\end{array}$ & 9 & 45,0 & 14 & 70,0 \\
\hline Não realiza & 11 & 55,0 & 6 & 28,56 \\
\hline Academia & 5 & 25,0 & 4 & 19,04 \\
\hline Musculação & 3 & 15,0 & 1 & 4,76 \\
\hline Pilates & 1 & 5,0 & 0 & 0,0 \\
\hline Futebol & 0 & 0,0 & 8 & 38,08 \\
\hline Corrida & 0 & 0,0 & 2 & 9,52 \\
\hline
\end{tabular}

Fonte: Dados da pesquisa 2018

$\mathrm{Na}$ tabela 3 destaca-se que os participantes apresentavam idades próximas, eram eutróficos e não apresentavam alterações patológicas nos valores antropométricos.

Tabela 3 - Caracterização da idade, antropometria, pressão arterial, FMR e FPP por sexo.

\begin{tabular}{|c|c|c|c|c|c|c|}
\hline \multirow[b]{2}{*}{ Variáveis } & \multicolumn{3}{|c|}{ Feminino $(n=20)$} & \multicolumn{3}{|c|}{ Masculino $(n=20)$} \\
\hline & Média & $\begin{array}{l}\text { Desvio } \\
\text { padrão }\end{array}$ & Mediana & Média & $\begin{array}{l}\text { Desvio } \\
\text { padrão }\end{array}$ & Mediana \\
\hline Idade (anos) & 23,80 & 5,28 & 21,50 & 21,25 & 2,73 & 21,00 \\
\hline Peso (kg) & 57,87 & 6,24 & 58,25 & 70,14 & 8,50 & 68,50 \\
\hline Altura (m) & 1,60 & 0,06 & 1,63 & 1,75 & 0,07 & 1,77 \\
\hline $\operatorname{IMC}\left(\mathrm{kg} / \mathrm{m}^{2}\right)$ & 22,41 & 2,08 & 22,70 & 22,77 & 1,71 & 23,30 \\
\hline Abdome (cm) & 73,95 & 5,77 & 74,00 & 80,25 & 7,12 & 80,00 \\
\hline Cintura (cm) & 72,30 & 6,53 & 72,50 & 78,55 & 6,53 & 79,00 \\
\hline Quadril (cm) & 97,10 & 6,43 & 98,00 & 98,15 & 5,94 & 98,50 \\
\hline $\mathrm{RCQ}(\mathrm{cm})$ & 0,73 & 0,06 & 0,74 & 0,79 & 0,04 & 0,79 \\
\hline PAS (mmHg) & 108,00 & 10,05 & 110,00 & 115,00 & 10,51 & 115,00 \\
\hline PAD (mmHg) & 74,50 & 8,25 & 80,00 & 74,50 & 8,87 & 80,00 \\
\hline
\end{tabular}




\begin{tabular}{lcccccc}
\hline FC (bpm) & 82,80 & 12,98 & 80,50 & 79,70 & 12,62 & 79,00 \\
PImax (cmH2O) & 98,00 & 32,86 & 90,00 & 120,00 & 50,67 & 100,00 \\
PEmax & 92,50 & 14,46 & 90,00 & 147,00 & 39,08 & 140,00 \\
(cmH2O) & 21,84 & 5,25 & 22,60 & 34,28 & 9,28 & 32,95 \\
FPP (kg/f) & & & & & \\
\hline
\end{tabular}

Fonte: Dados da pesquisa 2018.

No que diz respeito à correlação, observa-se na tabela 4 que houve correlação estatisticamente moderada entre PImax e PEmax no sexo feminino. Verifica-se, ainda, correlação estatisticamente fraca entre PImax e FPP no sexo masculino.

Tabela 4 - Correlação entre Pimax, Pemax e FPP divididas por sexo.

\begin{tabular}{|c|c|c|c|c|}
\hline \multicolumn{3}{|c|}{ Sexo } & Pimax & Pemax \\
\hline & Pemax & $\begin{array}{c}\text { Correlação de Pearson } \\
\text { Sig. (uni-caucal) }\end{array}$ & $\begin{array}{l}\mathbf{0 , 6 7} \\
<0,01\end{array}$ & \\
\hline Feminino & FPP & $\begin{array}{l}\text { Correlação de Pearson } \\
\text { Sig. (uni-caucal) }\end{array}$ & $\begin{array}{l}0,21 \\
0,19\end{array}$ & $\begin{array}{l}0,23 \\
0,16\end{array}$ \\
\hline & Pemax & $\begin{array}{c}\text { Correlação de Pearson } \\
\text { Sig. (uni-caucal) }\end{array}$ & $\begin{array}{l}0,32 \\
0,08\end{array}$ & \\
\hline Masculıno & FPP & $\begin{array}{l}\text { Correlação de Pearson } \\
\text { Sig. (uni-caucal) }\end{array}$ & $\begin{array}{r}-0,38^{*} \\
0,04\end{array}$ & $\begin{array}{l}0,25 \\
0,14\end{array}$ \\
\hline
\end{tabular}

Fonte: Dados da pesquisa 2018.

\section{DISCUSSÃO}

Este estudo se propôs investigar a associação da FPP e FMR em universitários. A partir das análises dos dados foi possível identificar médias maiores da FPP e FMR nos indivíduos do sexo masculino quando comparados com o sexo feminino.

Com relação à média de FPP e FMR obtida nos grupos avaliados, observouse um valor mais alto para o sexo masculino em relação ao sexo feminino em ambos os casos. Resultados semelhantes foram vistos no estudo de Tomás; Fernandes (2012), Angst et al., (2010) e Eichinger et al., (2015), os quais descreveram em suas 
pesquisas que os indivíduos do sexo masculino apresentavam valores de FPP mais elevado quando comparado aos indivíduos do sexo feminino, relatado também no estudo de Ferreira et al., (2011).

Almeida; Betucci; Lima (2008), em um estudo desenvolvido com indivíduos de ambos os sexos, para determinar FMR, após análise dos dados, verificou que os homens apresentaram a pressão inspiratória máxima (PImáx) e a pressão expiratória máxima (PEmáx) maiores em relação às mulheres, dados que coincidem com os resultados dessa pesquisa, onde as mulheres apresentaram valores de FMR menores em relação aos homens. Os autores apontam que esse fato pode estar relacionado aos comprimentos corporais e a diferença da força muscular entre os sexos. Em concordância Lima et al., (2016) justificam que o valor de FPP é mais forte nos homens em relação às mulheres, devido a massa muscular deles ser maior.

Nesta pesquisa, também foi observado que menos da metade das mulheres não tinha o hábito de praticar atividade física, enquanto a maioria dos homens praticava, justificando possivelmente os valores negativos da pesquisa, quanto a FPP e FMR nas mulheres.

Conforme Siqueira (2018) a prática exagerada de atividade física pode desencadear perda na capacidade muscular e, dessa forma, sobrecarregar a musculatura respiratória durante o exercício físico vigoroso. Os restos metabólicos do exercício físico, os quais devem ser removidos pelo corpo humano, se acumulam como resultado final da respiração celular, ocasionando, portanto, um aumento do trabalho respiratório no indivíduo, ou seja, significa dizer que ao respirar em um volume pulmonar maior, a ativação da musculatura inspiratória será menor. Tal redução poderá estar associada às consequentes diminuições da Pimax e as diferenças da FPP presentes nos participantes desta pesquisa do sexo masculino, de modo que eles autorrelataram na pesquisa que praticam atividade física por, pelo menos, 2 a 4 horas diariamente sem pausa.

Em contrapartida, Chaves et al. (2007) dizem que a prática de atividade física - como correr, caminhar, nadar, andar de bicicleta e jogar futebol - realizadas de maneira regular e moderada melhoram a capacidade de condução de oxigênio para os tecidos e órgãos, além de promover tolerância ao exercício e aumento da força 
muscular. Outro aspecto importante é que estes tipos de exercícios induzem o fortalecimento das fibras musculares, favorecendo o aumento da força da musculatura periférica e da musculatura respiratória.

Moreno et al. (2007) descrevem em sua literatura que o encurtamento da musculatura inspiratória pode ser um dos fatores desencadeadores da redução da Pimáx, isso ocorre em decorrência de alterações neuropsiquiátricas, tais como: estresse, hábitos posturais inadequados, fraqueza muscular, entre outros (NÓBREGA et al., 2019).

Agnol et al. (2018) e Coelho et al. (2012) afirmam que a Plmáx e PEmáx dependem uma da outra, tanto na força dos músculos respiratórios como no volume pulmonar, resultando em um equilíbrio na dinâmica respiratória. Fato observado entre as mulheres dessa pesquisa.

Após a análise dos resultados, observou-se que as médias da PImáx e PEmáx e FPP foram maiores nos homens em relação às mulheres. O mecanismo primário para essa redução, de acordo com alguns autores, é o aumento da idade, associado à diminuição da massa muscular, sedentarismo, e entre outros diferentes fatores capazes de influenciar nas mensurações.

Como limitação no presente estudo, ressalta-se a carência de dados na literatura sobre comparação do desempenho da FMR e da FPP em indivíduos saudáveis simultaneamente, visto que os artigos encontrados só comparam a FMR e a FPP em indivíduos que apresentam alguma patologia, puérperas ou associado à forma de tratamento, impossibilitando a análise comparativa com os resultados de outros autores. Outro aspecto a ser considerado, é o reduzido tamanho amostral, que pode ter limitado o poder da análise estatística. É importante ressaltar que novos estudos, com uma amostra maior e contendo indivíduos de várias etnias, idades e sexos, são necessários para que se possam obter novas correlações sobre a FMR e a força de preensão palmar. 


\section{CONCLUSÃO}

Observa-se que houve correlação estatisticamente significativa moderada entre PImax e PEmax no sexo feminino. Verifica-se, ainda, correlação estatisticamente fraca entre PImax e FPP no sexo masculino. Esse fato observado entre os participantes do sexo masculino pode ter ocorrido devido à prática de exercício físico específico, como o futebol, que aumenta a capacidade funcional e não trabalha de forma específica a musculatura respiratória. Já entre as mulheres a prática da musculação e a frequência em academia foi mais alta, de modo que os exercícios realizados podem ter contribuindo de forma indireta para o aumento da FMR. Entretanto, mais estudos com esse enfoque devem ser realizados para elucidar essas associações.

\section{REFERÊNCIAS BIBLIOGRÁFICAS}

AGNOL, S. M. D. et al. Comparação das pressões respiratórias máximas em idosos que praticam exercícios no solo e água. Revista Kairós: Gerontologia, v.20, n.2, p.399-41, 2018.

ALMEIDA, I. P; BERTUCCI, N. R; LIMA, V. P. de. Variações da pressão inspiratória máxima e pressão expiratória máxima a partir da capacidade residual funcional ou da capacidade pulmonar total e volume residual em indivíduos normais. O Mundo da Saúde, v. 32, n. 2, p. 176-82, 2008.

ANGST, Felix et al. Prediction of grip and key pinch strength in 978 healthy subjects. BMC musculoskeletal disorders, v. 11, n. 1, p. 94, 2010.

BESSA, E. J. C; LOPES, A J; RUFINO, R. A importância da medida da força muscular respiratória na prática da pneumologia. Pulmão RJ, v. 24, n. 1, p. 37-41, 2015.

BRASIL. Ministério da Saúde. Conselho Nacional de Saúde. Comissão Nacional de Ética em Pesquisa Resolução n. 466/12, de 12 de dezembro de 2012. Brasília: Ministério da Saúde, 2012.

CHAVES, C. R. M. D. M. et al. Exercício aeróbico, treinamento de força muscular e testes de aptidão física para adolescentes com fibrose cística: revisão da literatura, Rev. Bras. Saude Mater. Infant, v.7, n.3, p.245-250, 2007.

COELHO, C. M. et al. Comparação entre parâmetros de pressões respiratórias máximas em indivíduos saudáveis. Jornal Brasileiro de Pneumologia, v. 38, n.5, p.605-613, 2012. 
COSTA, D. et al. Novos valores de referência para pressões respiratórias máximas na população brasileira. Jornal Brasileiro Pneumologia, v. 36, n. 3, p. 306-12, 2010.

DIAS, J A. et al. Força de preensão palmar: métodos de avaliação e fatores que influenciam a medida. Revista Brasileira de Cineantropometria e desempenho Humano, v. 12, n. 3, p. 209-216, 2010.

EICHINGER, F. L. F. et al. Força de preensão palmar e sua relação com parâmetros antropométricos. Caderno da Terapia Ocupacional da UFScar, v. 23, n. 3, p. 525-532, 2015.

FERREIRA, A.C.C. et al. Força de preensão palmar e pinças em indivíduos sadios entre 6 e 19 anos. Acta Ortopédica Brasileira, v. 19, n. 2, p. 92-97, 2011.

FONSECA, M., C., S., M. S. Efeitos de programas de treinamento muscular respiratório na força muscular respiratória e na autonomia funcional de idosos. Memorialidades, v.13 n.25-26, p. 89118, 2017.

LIMA, T. R. et al. Força muscular em adultos e idosos jovens: associação com fatores sociodemográficos e estilo de vida. Dissertação (mestrado) - Universidade Federal de Santa Catarina, Centro de Ciências da Saúde, Programa de Pós-Graduação em Saúde Coletiva, Florianópolis, p.122, 2016.

MENDES, J; AZEVEDO, A; AMARAL, T. F. Força de preensão da mão: quantificação, determinantes e utilidade clínica. Arquivos de Medicina, Porto, v. 27, n. 3, p. 115-120, 2013.

MOREIRA, D. et al. Abordagem sobre preensão palmar utilizando o dinamômetro Jamar: uma revisão de literatura. Revista Brasileira de Ciência e Movimento, v. 11, n. 2, p. 95-100, 2008.

MORENO, M. A. et al. Efeito de um programa de alongamento muscular pelo método de Reeducação Postural Global sobre a força muscular respiratória e a mobilidade toracoabdominal de homens jovens sedentários. Jornal brasileiro de pneumologia, v. $33, n$. 6, p. 679-686, 2007.

NASCIMENTO, M.F. et al. Valores de referência de força de preensão manual em ambos os gêneros e diferentes grupos etários. Um estudo de revisão. EFDeportes. com, Revista Digital, n.151, 2010.

NÓBREGA, L.R. et al. Associação da força muscular respiratória e o estado nutricional de pacientes neurológicos. Revista Interdisciplinar em Saúde, v.6, n.1, p. 3-18, 2019.

REZENDE, F.A.C et al. Índice de Massa Corporal e Circunferência Abdominal: Associação com Fatores de risco Cardiovascular. Arquivos Brasileiro de Cardiologia. v.87, n.6, p.728-734, 2006.

SAMPAIO, L. R., FIGUEIREDO, V. D. C. Correlação entre o índice de massa corporal e os indicadores antropométricos de distribuição de gordura corporal em adultos e idosos. Revista de Nutrição, Campinas, v. 18, n.1, p. 53-6, 2005.

SANTOS, K. et al. Relação entre força muscular periférica e respiratória e qualidade de vida em pacientes com doenças pulmonar obstrutiva crônica. Medicina (Ribeirao Preto. Online), v. 48, n. 5, p. 417-424, 2015.

SIMÕES, R. P. et al. Influência da idade e do sexo na força muscular Respiratória. Fisioterapia e pesquisa, v. 14, n. 1, p. 36-41, 2007.

SIQUEIRA, V. D. S. Treinamento muscular respiratório: Uma abordagem teóricoprática no contexto da promoção da saúde. Trabalho de Conclusão Final de Curso. Mestrado Profissional em Exercício Físico na Promoção da Saúde. Universidade Norte do Paraná, 
Londrina, p.70, 2018.

SOARES, A. V. et al. Correlação entre os testes de dinamometria de preensão manual, escapular e lombar. Revista Acta Brasileira do Movimento Humano, Ji-Paraná, v. 2, n. 1, p. 65-72, 2012.

TOMÁS, M. T.; FERNANDES, M. B. Força De Preensão - Análise De Concordância Entre Dois Dinamómetros: Jamar Vs E-Link. Revista de Saúde e Tecnologia, v.7, p.39-43, maio 2012.

VALLE, P. H. C. et al. Efeitos do treinamento e destreinamento da força muscular respiratória em soldados. Revista Brasileira de Atividade Física \& Saúde, v. 7, n. 1, p. 46-54, 2012. 